\title{
Internal Symmetries of Differential Equations
}

\author{
Peter J. Olver ${ }^{\dagger}$ \\ School of Mathematics \\ University of Minnesota \\ Minneapolis, MN 55455 \\ U.S.A. \\ olver@math . umn . edu \\ http: //www. math. umn.edu/ olver
}

\begin{abstract}
Bäcklund's Theorem, which characterizes contact transformations, is generalized to give an analogous characterization of "internal symmetries" of systems of differential equations. For a wide class of systems of differential equations, every internal symmetry comes from a first order generalized symmetry and, conversely, every first order generalized symmetry satisfying certain explicit contact conditions determines an internal symmetry. We analyze the contact conditions in detail, deducing powerful necessary conditions for a system of differential equations admit "genuine" internal symmetries, i.e., ones which do not come from classical "external" symmetries. Applications include a direct proof that both the internal symmetry group and the first order generalized symmetries of a remarkable differential equation due to Hilbert and Cartan are the noncompact real form of the exceptional simple Lie group $G_{2}$.
\end{abstract}

The work I will survey in this paper, which will appear in [1], was done in collaboration with Ian Anderson, of Utah State University, and Niky Kamran, of McGill University. Our research had its genesis in a series of lectures on the variational bicomplex given by

$\dagger$ Supported in part by NSF Grant DMS 01-03944. 
Ian while visiting the University of North Carolina at Chapel Hill. Robert Bryant, who was in the audience, asked him to compute the symmetry group of the innocent looking underdetermined ordinary differential equation $u^{\prime}=\left(v^{\prime \prime}\right)^{2}$. Robert knew well the history of this equation, which we have decided to call the Hilbert-Cartan equation: Élie Cartan had proved that the "symmetry group" of this equation is isomorphic to the exceptional simple Lie group $G_{2}$ ! Robert was suitably impressed when Ian came back with a fourteen dimensional symmetry algebra for the equation. There matters rested until, during a Conference on Symbolic Manipulation hosted by the I.M.A. in Minnesota, Robby Gardner asked Fritz Schwarz to answer the same question using his computer algebra package for computing symmetry groups in SCRATCHPAD (now renamed AxIOM). Fritz only found a six-dimensional symmetry group. After Ian sent the results of his earlier (hand!) computations, we realized that the discrepancy was due to the fact that Ian had computed the first order generalized symmetries of the equation, whereas Fritz' program was designed to compute classical point symmetries, which is why he failed to detect the eight remaining vector fields. However, upon reflection, it occurred to us that much more was at stake than merely the difference between point symmetries and generalized symmetries. As far as we know, Cartan was not aware of the concept of a generalized symmetry, and all his symmetries were realized as geometrical transformations of some finite-dimensional space, which the generalized symmetries are not. Contact transformations fit into Cartan's framework, but these were not the objects Cartan had computed for this particular equation since, according to Bäcklund's Theorem, there are no contact transformations (beyond prolonged point transformations) if the number of dependent variables is greater than one. What Cartan had computed were what we will call "internal symmetries", which are transformations which preserve the contact ideal only when restricted to the equation submanifold. (These are also known as "dynamical symmetries" in the mathematical physics literature, and have also received mention in the work of Vinogradov and his collaborators, cf. [10].) The restrictions of Bäcklund's Theorem no longer apply, and there are internal symmetries which depend explicitly on higher order derivatives. Thus, a new question arose: for the Hilbert-Cartan equation, why did the computed Lie algebra of generalized symmetries coincide with Cartan's Lie algebra of internal symmetries?

Our results answer this question in general, and can be summarized as follows. First, and obvious, is the fact that every external symmetry restricts to an internal symmetry. In many cases, including all normal systems of partial (not ordinary) differential equations of order at least two, all internal symmetries arise in this way. Second, under a certain condition on the system of differential equations, which we name the "descent property", every internal symmetry comes from a first order generalized symmetry, a result that significantly ameliorates the computation of these symmetries. The systems covered by this result include all second order systems of differential equations, all normal systems of partial differential equations, and a wide class of higher order underdetermined ordinary differential equations. Our theorem includes the classical Bäcklund Theorem as a particular special case. Finally, every first order generalized symmetry which satisfies additional contact conditions is equivalent to an internal symmetry. In certain cases, such as the "codimension 1" ordinary differential equations, of which the Hilbert-Cartan equation is a particular example, there are no contact restrictions, hence there is a one-to-one 
correspondence between internal symmetries and first order generalized symmetries.

In order to keep the exposition as brief as possible, I will assume that the reader is familiar with the standard theory of symmetry groups of differential equations as presented, for instance, in [12]. We will work with local coordinates throughout, although all of the results have analogous, more general, statements in the context of fiber bundles over smooth manifolds. Consider a system of differential equations

$$
\mathcal{R}: \quad \Delta_{\kappa}\left(x, u^{(n)}\right)=0, \quad \kappa=1, \ldots, r,
$$

in $p$ independent variables $x=\left(x^{1}, \ldots, x^{p}\right)$, and $q$ dependent variables $u=\left(u^{1}, \ldots, u^{q}\right)$. Derivatives $u_{J}^{\alpha}=\partial^{J} u^{\alpha} / \partial x^{J}$ of the dependent variables of order $k=\# J$ are indexed by symmetric multi-indices $J=\left(j_{1}, \ldots, j_{k}\right), 1 \leq j_{\nu} \leq p$. We let $u^{(n)}$ denote the collection of all such derivatives of orders $k \leq n$, which provide coordinates on the associated jet space $\mathrm{J}^{n}$, so that the system (1) determines a subvariety $\mathcal{R} \subset \mathrm{J}^{n}$. Let $D_{i}$ denotes the total derivative with respect to $x^{i}$, and $D_{J}=D_{j_{1}} \cdots D_{j_{k}}$ the corresponding $k^{\text {th }}$ order total derivative, so that $u_{J}^{\alpha}=D_{J} u^{\alpha}$. The $k^{\text {th }}$ order prolongation of the $n^{\text {th }}$ order system of differential equations $(1)$ is the $(n+k)^{\text {th }}$ order system obtained by (totally) differentiating the equations in $\mathcal{R}$ up to $k$ times:

$$
\operatorname{pr}^{(k)} \mathcal{R}: \quad D_{J} \Delta_{\kappa}\left(x, u^{(n+k)}\right)=0, \quad \kappa=1, \ldots, r, \quad \# J \leq k,
$$

so that $\operatorname{pr}^{(k)} \mathcal{R} \subset \mathrm{J}^{n+k}$ is a subvaritey of the $(n+k)^{\text {th }}$ order jet space. We will assume that the system (1) and all its prolongations (2) satisfy the mild nondegeneracy conditions of being locally solvable, cf. $[\mathbf{1 2} ; \S 2.6]$, and that its Jacobian matrix with respect to the $n^{\text {th }}$ order derivatives of $u$ is of maximal rank; this implies that the system and its prolongations define regular submanifolds $\mathrm{pr}^{(k)} \mathcal{R} \subset \mathrm{J}^{n+k}$ of the jet space. The top order maximal rank condition is slightly stronger than the standard maximal rank condition, [12].

In general, by a symmetry of the system of differential equations (1) we mean a transformation which maps solutions to solutions. The most basic type of symmetry is a point transformation, meaning a local diffeomorphism $\Phi:(x, u) \longmapsto(\bar{x}, \bar{u})$ on the space $M$ of independent and dependent variables. A connected local group of point transformations $G$ is generated, via the usual process of exponentiation, by a Lie algebra of vector fields

$$
\mathbf{v}=\sum_{i=1}^{p} \xi^{i}(x, u) \frac{\partial}{\partial x^{i}}+\sum_{\alpha=1}^{q} \varphi^{\alpha}(x, u) \frac{\partial}{\partial u^{\alpha}},
$$

on the space of independent and dependent variables. The group transformations $g \in G$ act on functions $u=f(x)$ by pointwise transforming their graphs, and hence they also act on their derivatives. This action induces the prolonged group action $\operatorname{pr}^{(n)} G$ on the jet space $\mathrm{J}^{n}$, whose explicit transformation rules are very complicated. However the corresponding prolonged infinitesimal generators have a rather simple "prolongation formula"

$$
\operatorname{pr}^{(n)} \mathbf{v}=\sum_{i=1}^{p} \xi^{i}(x, u) \frac{\partial}{\partial x^{i}}+\sum_{\alpha=1}^{q} \sum_{\# J=j=0}^{n} \varphi_{J}^{\alpha}\left(x, u^{(j)}\right) \frac{\partial}{\partial u_{J}^{\alpha}}
$$


which is the vector field on the jet space $\mathrm{J}^{n}$ whose coefficients $\varphi_{J}^{\alpha}$ are determined via the well-known recursive formula

$$
\varphi_{J, i}^{\alpha}=D_{i} \varphi_{J}^{\alpha}-\sum_{j=1}^{p} D_{i} \xi^{j} u_{J, j}^{\alpha}
$$

Theorem 1. Assume that the system of partial differential equations (1) is nondegenerate. Then the vector field $\mathbf{v}$ in (3) will generate a one-parameter symmetry group of the system (1) if and only if the classical infinitesimal symmetry criterion holds:

$$
\operatorname{pr}^{(n)} \mathbf{v}\left(\Delta_{\nu}\right)=0, \quad \text { whenever } \quad \Delta=0, \quad \nu=1, \ldots, r .
$$

The "determining equations" (6) form a large over-determined linear system of partial differential equations for the coefficients $\xi^{i}, \varphi^{\alpha}$ of $\mathbf{v}$, and can, in practice, be explicitly solved to determine the complete (connected) symmetry group of the system (1). There are now a wide variety of computer algebra packages available which will automate most of the routine steps in the calculation of the symmetry group of a given system of partial differential equations. See $[\mathbf{4}],[5]$, for useful surveys of the different packages available, including discussions of their strengths and weaknesses.

The theory of point symmetries of differential equations is classical, and, in more or less the same form, dates back to the original work of Sophus Lie. After this theory is well understood, a number of possible generalizations come to mind. The first direction, originally taken by E. Noether, [11], is to allow generalized vector fields

$$
\mathbf{v}=\sum_{i=1}^{p} \xi^{i}\left(x, u^{(k)}\right) \frac{\partial}{\partial x^{i}}+\sum_{\alpha=1}^{q} \varphi^{\alpha}\left(x, u^{(k)}\right) \frac{\partial}{\partial u^{\alpha}},
$$

whose coefficients can also depend on derivatives of $u$. The condition that $\mathbf{v}$ be a generalized symmetry of the system of differential equations (1) is the same as before, (6), although now one must also take into account the prolongations (2) of the system. Every generalized symmetry is equivalent to one in evolutionary form

$$
\mathbf{v}_{Q}=\sum_{\alpha=1}^{q} Q^{\alpha}\left(x, u^{(1)}\right) \frac{\partial}{\partial u^{\alpha}}
$$

where the $q$-tuple of functions $Q=\left(Q^{1}, \ldots, Q^{q}\right)$, known as the characteristic of $\mathbf{v}$, has entries

$$
Q^{\alpha}\left(x, u^{(k)}\right)=\varphi^{\alpha}\left(x, u^{(k)}\right)-\sum_{i=1}^{p} \xi^{i}\left(x, u^{(k)}\right) \frac{\partial u^{\alpha}}{\partial x^{i}}, \quad \alpha=1, \ldots, q .
$$

Replacing the generalized vector field $\mathbf{v}$ by its evolutionary form $\mathbf{v}_{Q}$ leads to a simpler set of determining equations in that they only involve the $q$ unknown functions $Q^{\alpha}$ rather than the $p+q$ unknown coefficients $\xi^{i}, \varphi^{\alpha}$ of $\mathbf{v}$. (This technique even works for point symmetries, where the associated characteristic depends linearly on first order derivatives.) An evolutionary vector field $\mathbf{v}_{Q}$ is a trivial symmetry of the system (1) if the characteristic $Q$ vanishes on all solutions. Two generalized symmetries $\mathbf{v}$ and $\mathbf{w}$ are equivalent if their 
respective evolutionary forms differ by a trivial evolutionary symmetry. The flow generated by an evolutionary symmetry is nonlocal, being found by solving the system of evolution equations $u_{t}^{\alpha}=Q^{\alpha}\left(x, u^{(n)}\right), \alpha=1, \ldots, q$.

A $k^{\text {th }}$ order generalized vector field is will not usually prolong to a well-defined vector field on any jet bundle $\mathrm{J}^{n}$ since its $n^{\text {th }}$ prolongation will involve derivatives of orders up to $k+n$. Beyond point transformations, the only exceptions to this are the infinitesimal contact transformations. Given a function $u=f(x)$, let $u^{(n)}=\mathrm{pr}^{(n)} f(x)$ denote its $n$-jet or $n^{\text {th }}$ prolongation. The graph $\Gamma_{f}^{(n)}$ of $\operatorname{pr}^{(n)} f$ is a $p$-dimensional submanifold of $\mathrm{J}^{n}$. A one-form $\theta$ on $\mathrm{J}^{n}$ is called a contact form if it restricts to zero on every prolonged graph $\Gamma_{f}^{(n)}$. It is not hard to prove that every contact form is a linear combination of the basic contact one-forms

$$
\theta_{J}^{\alpha}=d u_{J}^{\alpha}-u_{J, i}^{\alpha} d x^{i}, \quad \alpha=1, \ldots, q, \quad 0 \leq \# J<n,
$$

which generate the contact ideal $\mathcal{I}^{(n)}$ on $\mathrm{J}^{n}$. Conversely, any $p$-dimensional submanifold $\Gamma \subset \mathrm{J}^{n}$ which is transversal to the vertical directions, and annihilates the contact ideal $\mathcal{I}^{(n)} \mid \Gamma=0$, is (locally) the graph of prolonged function $u^{(n)}=\operatorname{pr}^{(n)} f(x)$. (Here $\mid \Gamma$ denotes the pull-back to the submanifold $\Gamma$, i.e., if $\iota: \Gamma \rightarrow \mathrm{J}^{n}$ is the natural embedding, then $\mathcal{I}^{(n)} \mid \Gamma=\iota^{*}\left(\mathcal{I}^{(n)}\right)$.) A (locally defined) transformation $\Psi: \mathrm{J}^{n} \rightarrow \mathrm{J}^{n}$ on the jet space will determine a contact transformation provided its pull-back $\Psi^{*}$ maps contact forms to contact forms, which means that it preserves the contact ideal:

$$
\Psi^{*}\left(\mathcal{I}^{(n)}\right) \subset \mathcal{I}^{(n)}
$$

The infinitesimal version of this criterion is that a vector field

$$
\mathbf{X}=\sum_{i=1}^{p} \xi^{i}\left(x, u^{(n)}\right) \frac{\partial}{\partial x^{i}}+\sum_{\alpha=1}^{q} \sum_{\# J=0}^{n} \varphi_{J}^{\alpha}\left(x, u^{(n)}\right) \frac{\partial}{\partial u_{J}^{\alpha}},
$$

on $\mathrm{J}^{n}$ generates a one-parameter group of contact transformations provided the Lie derivative of any contact form is contained in the contact ideal:

$$
\mathbf{X}\left[\theta_{K}^{\alpha}\right]=\sum_{\beta=1}^{q} \sum_{\# J=0}^{\# K} \mu_{K, \beta}^{\alpha, J} \theta_{J}^{\beta}, \quad \alpha=1, \ldots, q, \quad \# K<n,
$$

for functions $\mu_{K, \beta}^{\alpha, J}: J^{n} \rightarrow \mathbb{R}$. These conditions are quite restrictive, as shown by the classical theorem due to Bäcklund.

Theorem 2. If the number of dependent variables is more than one, $q>1$, then every contact transformation on $\mathrm{J}^{n}$ is the $n^{\text {th }}$ prolongation of a point transformation. If there is a single dependent variable, $q=1$, then every contact transformation on $\mathrm{J}^{n}$ is the $(n-1)^{\text {st }}$ prolongation of a first order contact transformation on $\mathrm{J}^{1}$.

The projection

$$
\pi(\mathbf{X})=\sum_{i=1}^{p} \xi^{i}\left(x, u^{(1)}\right) \frac{\partial}{\partial x^{i}}+\sum_{\alpha=1}^{q} \varphi^{\alpha}\left(x, u^{(1)}\right) \frac{\partial}{\partial u^{\alpha}},
$$


of any contact vector field gives a first order generalized vector field, or, if $q>1$, of a point vector field, as in (3). The next lemma is utilized to provide a characterization of which generalized vector fields produce contact transformations. As such, it plays a key role in the standard infinitesimal proof of Bäcklund's Theorem 2, cf. [7].

Lemma 3. An evolutionary vector field $\mathbf{v}_{Q}$ is equivalent to an infinitesimal contact transformation if and only if its characteristic $Q\left(x, u^{(1)}\right)$ depends on at most first order derivatives, and there exist functions $\xi^{i}\left(x, u^{(1)}\right), i=1, \ldots, p$, such that the following contact conditions hold:

$$
\frac{\partial Q^{\alpha}}{\partial u_{i}^{\beta}}+\xi^{i} \delta_{\beta}^{\alpha}=0, \quad \alpha, \beta=1, \ldots, q, \quad j=1, \ldots, p .
$$

Indeed, in this case, each $\xi^{i}$ will be the coefficient of $\partial / \partial x i$ in the projection $\mathbf{v}=\pi(\mathbf{X})$, cf. (14), and the coefficients of the $\partial / \partial u^{\alpha}$ will be defined by

$$
\varphi^{\alpha}=Q^{\alpha}+\sum_{i=1}^{p} \xi^{i} u_{i}^{\alpha}, \quad \alpha=1, \ldots, q .
$$

The contact vector field $\mathbf{X}$ is then just the $n^{\text {th }}$ prolongation of its projection $\mathbf{v}=\pi(\mathbf{X})$. Note that left hand sides of the contact conditions (15) appear in the prolongation formula as the coefficients of the terms in $\mathrm{pr}^{(n)} \mathbf{v}$ which depend on derivatives of order $n+1$, hence their vanishing is a necessary and sufficient condition that the prolongation $\operatorname{pr}^{(n)} \mathbf{v}$ of the first order generalized vector field (16) define a genuine vector field on $\mathrm{J}^{n}$.

In the case of one dependent variable, $q=1$, there are no Greek indices in the contact conditions (15), and so these equations serve to define the coefficients $\xi^{i}$. Thus, any first order generalized symmetry will give rise to a contact transformation. Indeed, the characteristic $Q\left(x, u^{(1)}\right)$ can be identified with the negative of Lie's characteristic function (hence the name) which generates the one-parameter group of contact transformations. For more than one dependent variable, $q>1$, the integrability conditions for the system of partial differential equations (15) will require that $\xi^{i}, \varphi^{\alpha}$ depend only on $x$, $u$, and so every contact transformation reduces to a point transformation.

We shall call a group of contact transformations which preserves a given system of differential equations an external symmetry group as the transformations are defined on open subsets of the the jet space $\mathrm{J}^{n}$, and can thereby be used to transform arbitrary functions $u=f(x)$. Thus any external symmetry group of a system of differential equations is characterized by two conditions: $a$ ) it leaves the equation submanifold $\mathcal{R}$ invariant, and b) it preserves the contact ideal on $\mathrm{J}^{n}$. Bäcklund's Theorem 2 implies that the second condition is very restrictive and severely limits the possible geometrical symmetries beyond point transformations. However, since we are only really interested in what the symmetry group does to solutions of the system of differential equations, and thus in its restriction to the equation submanifold $\mathcal{R}$, it makes sense to relax the second condition and only require that the group transformations preserve the contact ideal on $\mathcal{R}$, rather than all of $\mathrm{J}^{n}$.

Definition 4. An internal symmetry of a system of differential equations $\mathcal{R} \subset \mathrm{J}^{n}$ is an invertible transformation $\Psi: \mathcal{R} \rightarrow \mathcal{R}$ which maps $\mathcal{R}$ to itself and which preserves the 
restriction of the contact ideal on $\mathcal{R}$ :

$$
\Psi^{*}\left(\mathcal{I}^{(n)} \mid \mathcal{R}\right) \subset \mathcal{I}^{(n)} \mid \mathcal{R}
$$

An internal symmetry will, consequently, transform solutions of the system to solutions. Clearly any external symmetry restricts to an internal symmetry, but it is not necessarily true that an internal symmetry can be extended off the solution manifold to a genuine contact transformation. Indeed, Bäcklund's Theorem in its original form no longer applies to internal symmetries, and, as we shall see, there are $n^{\text {th }}$ order internal symmetries which are not the prolongation of any lower order contact map. However, every internal symmetry can be viewed as a particular type of generalized symmetry, and so internal symmetries are seen to occupy a position intermediate to external and generalized symmetries. They form the widest possible class of symmetries which can be realized as local geometrical transformations on a submanifold of jet space.

In the case of continuous groups of internal symmetries, we can again work infinitesimally. Let $\mathbf{X}$ be a vector field on the submanifold $\mathcal{R}$, which, in local coordinates, takes the form (12) above, where the coefficients $\xi^{i}, \varphi_{J}^{\alpha}$ are now only need be defined on $\mathcal{R}$, although we may always assume, without essential loss of generality, that we have extended the vector field off the submanifold, the precise extension not being important. The infinitesimal symmetry condition is that $\mathbf{X}$ is tangent to $\mathcal{R}$, which, in local coordinates, says

$$
\mathbf{X}\left(\Delta_{\nu}\right)=0, \quad \nu=1, \ldots, r, \quad \text { whenever } \quad \Delta=0
$$

in direct analogy with (6). In addition, $\mathbf{X}$ must preserve the contact ideal on $\mathcal{R}$ :

$$
\mathbf{X}\left(\mathcal{I}^{(n)} \mid \mathcal{R}\right) \subset \mathcal{I}^{(n)} \mid \mathcal{R}
$$

Note that the projection $\mathbf{v}=\pi(\mathbf{X})$, cf. (14), of any internal symmetry determines an $n^{\text {th }}$ order generalized vector field. (The coefficients $\xi^{i}, \varphi^{\alpha}$, are a priori only defined on $\mathcal{R}$, but the projections of two different extensions of $\mathbf{X}$ will differ only by a trivial generalized symmetry.) It is not difficult to see that $\mathbf{v}$ is a generalized symmetry of the system whose prolongation agrees with $\mathbf{X}$ when restricted to the system. Now, in general, $\mathbf{X}$ and $\mathbf{v}$ will depend on $n^{\text {th }}$ order derivatives of the $u$ 's. However, under certain conditions on the system of differential equations, we can prove every internal symmetry $\mathbf{X}$ has a characteristic $Q$ which depends on at most first order derivatives.

Definition 5. Let $n \geq 3$. An $n^{\text {th }}$ order system of differential equations $\mathcal{R}$ is said to have the descent property if it satisfies the following: Suppose $Q\left(x, u^{(n-1)}\right)$ is a smooth function of order $n-1$ such that all of its total derivatives $D_{1} Q, \ldots, D_{p} Q$, when restricted to the system, also have order $n-1$. Then $Q\left(x, u^{(n-2)}\right)$ must have of order $n-2$.

In other words, if $\mathcal{R}$ has the descent property, then the only functions whose total derivatives, restricted to the system, have order $n-1$ are those of order $n-2$. First and second order systems have the descent property by default. It is easy to see that open subsets of $\mathrm{J}^{n}$ have the descent property, as do normal (Kovalevskaya) systems of partial differential equations in $p>1$ independent variables. We can now state the main result relating internal symmetries and generalized symmetries. 
Theorem 6. If $\mathcal{R}$ is a nondegenerate system of differential equations satisfying the descent property, then every internal symmetry is equivalent to a first order generalized symmetry in evolutionary form.

A normal system of ordinary differential equations has the standard form

$$
u_{n}^{\alpha}=F^{\alpha}\left(x, u^{(n-1)}\right), \quad \alpha=1, \ldots, q,
$$

in which there are the same number of equations as unknowns, and we have solved for the top order derivatives. Successively differentiating (20), shows that we can re-express all $n^{\text {th }}$ and higher order derivatives of $u$ in terms of those of order at most $n-1$, and so the descent property clearly does not hold if $n \geq 3$. This also immediately implies that every generalized symmetry of (20) determines an internal symmetry, but there is no reason that the generalized symmetry have order one. Indeed the trivial scalar equation $u_{x x x}=0$ has second order internal symmetry $u_{x x} \partial_{u}$ which clearly has no first order counterpart.

Let us now investigate the conditions that a first order generalized symmetry must satisfy in order that it determine an internal symmetry. The contact conditions (19) take the form

$$
\mathbf{X}\left[\theta_{K}^{\alpha}\right]=\sum_{\beta, J} \mu_{K, \beta}^{\alpha, J} \theta_{J}^{\alpha}+\sum_{\kappa} \lambda_{K}^{\alpha, \kappa} d \Delta_{\kappa}, \quad \text { on } \quad \mathcal{R}, \quad \alpha=1, \ldots, q, \quad \# J<n,
$$

in analogy with (13). Here the $\mu_{K, \beta}^{\alpha, J}, \lambda_{K}^{\alpha, \kappa}$ are functions on $\mathcal{R}$, and by the phrase "on $\mathcal{R}$ " we mean that the individual coefficients of the basis one-forms $d x^{i}, d u_{K}^{\alpha}$ must agree when restricted to the submanifold $\mathcal{R}$. (The fact that the pull-backs of these one-forms to $\mathcal{R}$ are no longer linearly independent has been taken care of by the $\lambda_{K}^{\alpha, \kappa}$, which play the role of Lagrange multipliers.) Detailed analysis of (21) shows that the coefficients of X must satisfy the prolongation formula (5) modulo the system and its derivatives:

$$
\varphi_{J, i}^{\alpha}=D_{i} \varphi_{J}^{\alpha}-\sum_{j=1}^{p} D_{i} \xi^{j} u_{J, j}^{\alpha} \quad \text { on } \quad \operatorname{pr}^{(n)} \mathcal{R} .
$$

Moreover, there is an additional set of "internal contact conditions" analogous to (15), arising from the fact that, on the equation submanifold, the $n^{\text {th }}$ order terms arising from the restricted prolongation formula $(22)$ cannot depend on $(n+1)^{\text {st }}$ order derivatives. In order to write them in a reasonably compact form, we introduce some additional auxiliary (complex) variables $\zeta=\left(\zeta_{1}, \ldots, \zeta_{p}\right)$, and define the monomial $\zeta_{K}=\zeta_{k_{1}} \zeta_{k_{2}} \cdots \zeta_{k_{n}}$ when $K=\left(k_{1}, \ldots, k_{n}\right)$ is a symmetric multi-index. The $r \times q$ matrix of homogeneous polynomials of degree $n$ in $\zeta$ given by

$$
\mathbf{D}(\zeta)=\left(\sum_{\# K=n} \frac{\partial \Delta_{\kappa}}{\partial u_{K}^{\beta}} \zeta_{K}\right),
$$

plays a key role in the definition of the classical characteristic directions (not to be confused with the characteristic $Q$ !) for the system of partial differential equations (1). For example, assume that $r=q$, so we have the same number of equations as unknowns. A 
complex direction $\zeta$, which should be thought of as defining coordinates in the complexified cotangent bundle $T_{\mathbb{C}}^{*} X=T^{*} X \otimes \mathbb{C}$ of the independent variable space $X$, determines a characteristic direction at the point $\left(x, u^{(n)}\right) \in \mathcal{R}$ if and only if $\operatorname{det} \mathbf{D}(\zeta)=0$. A system is called normal if not every direction is characteristic, i.e., $\operatorname{det} \mathbf{D}(\zeta) \not \equiv 0$; this is equivalent to the existence of local coordinates in which the system assumes a form amenable to the application of the Cauchy-Kovalevskaya existence theorem, cf. [12; Theorem 2.79]. Further, given the characteristic $Q\left(x, u^{(1)}\right)$ of a first order generalized symmetry, we define the analogous $q \times q$ matrix of homogeneous linear polynomials

$$
\mathbf{R}(\zeta)=\left(\sum_{j=1}^{p} \frac{\partial Q^{\alpha}}{\partial u_{j}^{\beta}} \zeta_{j}\right)
$$

Theorem 7. Let $\mathbf{v}_{Q}$ be a first order generalized symmetry of a nondegenerate system of partial differential equations $\mathcal{R}$. Then there is an internal symmetry $\mathbf{X}$ with evolutionary form $\mathbf{v}_{Q}$ if and only if there exist functions $\xi^{1}\left(x, u^{(n)}\right), \ldots, \xi^{p}\left(x, u^{(n)}\right)$, defined on $\mathcal{R}$, such that, for every homogeneous scalar polynomial $P(\zeta)$ of degree $n$, there exists an $q \times r$ matrix of linear polynomials $\mathbf{L}_{P}(\zeta)$ (which can depend on both the polynomial $P$ and the point $\left.\left(x, u^{(n)}\right) \in \mathcal{R}\right)$ satisfying the internal contact conditions

$$
P(\zeta)\{\mathbf{R}(\zeta)+(\xi \cdot \zeta) \mathbf{I}\}=\mathbf{L}_{P}(\zeta) \cdot \mathbf{D}(\zeta) \quad \text { on } \quad \mathcal{R} .
$$

In this matrix equation, $\mathbf{I}$ denotes the $q \times q$ identity matrix, and $\xi \cdot \zeta=\sum_{i=1}^{p} \xi^{i} \zeta_{i}$.

If the internal contact conditions (25) are satisfied, then the internal symmetry $\mathbf{X}$ associated with the evolutionary symmetry $\mathbf{v}_{Q}$ is the $n^{\text {th }}$ prolongation of the equivalent generalized vector field $\mathbf{v}$ as in (7), whose coefficients $\xi^{i}, \varphi^{\alpha}$, are related to $Q$ via (16). The internal contact conditions (25) guarantee that, on the equation submanifold $\mathcal{R}$, the $n^{\text {th }}$ prolongation of $\mathbf{v}$ does not depend on $(n+1)^{\text {st }}$ order derivatives, and so defines a genuine internal symmetry; see the remarks following Lemma 3. Also note that, even though $\mathbf{v}_{Q}$ is a first order generalized vector field, the equivalent generalized vector field $\mathbf{v}$ can have order $n$ since the functions $\xi^{i}$ which satisfy (25) may depend on higher order derivatives.

In order to understand what these conditions mean more concretely, we discuss some particular examples. First consider the extreme case in which there are no differential equations, i.e., the equation submanifold $\mathcal{R}$ is an open subset of $\mathrm{J}^{n}$. In this degenerate case, the right hand side of the contact conditions (25) is automatically zero, and so the polynomial $P(\zeta)$ can be ignored. The resulting condition

$$
\mathbf{R}(\zeta)+(\xi \cdot \zeta) \mathbf{I}=0
$$

are easily seen to be the same as the contact conditions (15) for ordinary contact transformations - an "internal symmetry of $\mathrm{J}^{n}$ " just means an ordinary contact transformation.. Thus, in this case, Theorem 6 reduces to the classical Theorem of Bäcklund that every contact transformation comes from a first order contact transformation, and we are justified in labelling Theorem 6 as a generalization of Bäcklund's Theorem 2 to systems of differential equations. 
In many cases, the contact conditions (25) will be so restrictive as to automatically imply that the left hand side must vanish. Indeed, the $q \times q$ matrix

$$
\mathbf{M}(\zeta)=\mathbf{R}(\zeta)+(\xi \cdot \zeta) \mathbf{I}
$$

of linear functions of $\zeta$ measures, in a sense, the "degree of internality" of the symmetry $\mathbf{v}_{Q}$. More specifically, according to Lemma 3, an internal symmetry with first order characteristic will extend to an external (contact) symmetry if and only if the corresponding matrix $\mathbf{M}(\zeta)$ is identically zero for some choice of functions $\xi^{i}$. Many systems of partial differential equations do not have any non-extendable internal symmetries, and the contact conditions (25) are an effective means of detecting this. For example:

Theorem 8. If $\mathcal{R}$ is a normal system of partial differential equations in $p \geq 2$ independent variables, of order $n \geq 2$, then every internal symmetry extends to an external symmetry.

For an $n^{\text {th }}$ order system of ordinary differential equations,

$$
\Delta_{\kappa}\left(x, u^{(n)}\right)=0, \quad \kappa=1, \ldots, r,
$$

the internal contact conditions (25) dramatically simplify. Since $\zeta \in \mathbb{C}$, the polynomial $P(\zeta)$ is a multiple of $\zeta^{n}$, so $\zeta$ can be eliminated entirely. Let

$$
\mathbf{D}=\left(\frac{\partial \Delta_{\kappa}}{\partial u_{n}^{\beta}}\right), \quad \mathbf{R}=\left(\frac{\partial Q^{\alpha}}{\partial u_{x}^{\beta}}\right),
$$

where $u_{n}^{\beta}=d^{n} u^{\beta} / d x^{n}$, be, respectively, $r \times q$ and $q \times q$ matrices depending on $\left(x, u^{(n)}\right)$ and $\left(x, u^{(1)}\right)$. The internal contact conditions $(25)$ reduce to

$$
\mathbf{R}+\xi \mathbf{I}=\mathbf{L} \cdot \mathbf{D} \quad \text { on } \quad \mathcal{R},
$$

for some $q \times r$ matrix $\mathbf{L}$. Writing (30) out in components, we require

$$
\frac{\partial Q^{\alpha}}{\partial u_{x}^{\beta}}+\xi \delta_{\beta}^{\alpha}=\sum_{\kappa=1}^{r} \lambda_{\kappa}^{\alpha} \frac{\partial \Delta_{\kappa}}{\partial u_{n}^{\beta}}, \quad \text { on } \quad \mathcal{R}, \quad \alpha, \beta=1, \ldots, q,
$$

for some unspecified functions $\xi, \lambda_{\kappa}^{\alpha}$. As before, the internal symmetry associated with $\mathbf{v}_{Q}$ is given by $\mathbf{X}=\operatorname{pr}^{(n)} \mathbf{v}$, where coefficients of $\mathbf{v}$ are related to $Q$ via (16).

It is not always necessary to verify all of the contact conditions (31), as some of them are direct consequences of the symmetry conditions. A system (28) is said to be of codimension $c=q-r$ if the $q \times r$ Jacobian matrix $\mathbf{D}$ has maximal rank $r \leq q$. The implicit function theorem assures us that we can locally solve (28) for $r$ of the top order derivatives, say $u_{n}^{1}, \ldots, u_{n}^{r}$, which results in a system of ordinary differential equations of the form

$$
u_{n}^{\kappa}=\Gamma^{\kappa}\left(x, u^{(n-1)}, u_{n}^{r+1}, \ldots, u_{n}^{q}\right), \quad \kappa=1, \ldots, r .
$$

With this choice, we will refer to the variables $u^{1}, \ldots, u^{r}$, as normal directions, and the variables $u^{r+1}, \ldots, u^{q}$, as tangential directions. Although normal systems of ordinary differential equations of order $n \geq 3$ do not satisfy the descent property, most of the 
underdetermined systems $(32)$ do. Let $\mathbf{H}$ denote the $\frac{1}{2}(q-r)(q-r+1) \times r$ tangential Hessian matrix with entries $\partial^{2} \Gamma^{\kappa} / \partial u_{n}^{\lambda} \partial u_{n}^{\mu}$, with rows indexed by $\lambda, \mu=r+1, \ldots, q$, and columns indexed by $\kappa=1, \ldots, r$.

Proposition 9. The system (32) has the descent property if its tangential Hessian matrix $\mathbf{H}$ has (maximal) rank $r$.

Theorem 10. Let $\mathcal{R}$ be an $n^{\text {th }}$ order system of ordinary differential equations of codimension $c=q-r$. A first order generalized symmetry $\mathbf{v}_{Q}$ is equivalent to an internal symmetry $\mathbf{X}$ if and only if there exist functions $\xi\left(x, u^{(n)}\right)$, and $\lambda_{\kappa}^{\alpha}\left(x, u^{(n)}\right), \kappa=1, \ldots, r$, $\alpha=r+1, \ldots, q$, defined on $\mathcal{R}$, satisfying the tangential contact conditions

$$
\frac{\partial Q^{\alpha}}{\partial u_{x}^{\beta}}+\xi \delta_{\beta}^{\alpha}=\sum_{\kappa=1}^{r} \lambda_{\kappa}^{\alpha} \frac{\partial \Delta_{\kappa}}{\partial u_{n}^{\beta}}, \quad \beta=1, \ldots, q, \quad \text { on } \quad \mathcal{R}, \quad \alpha=r+1, \ldots, r .
$$

Indeed, the remaining normal contact conditions - equations (31) for $\alpha=1, \ldots, r$, $\beta=1, \ldots, q-$ are found to be direct consequences of the tangential contact conditions and the symmetry conditions. In particular, if the system has the descent property, then Theorem 10 provides a one-to-one correspondence between internal symmetries and first order generalized symmetries which satisfy the contact conditions in the tangential directions.

If a system has codimension 1 , then $\mathbf{D}$ has rank $q-1$. There is just one tangential direction, say $u^{q}$, and so the tangential contact conditions (33) for $\alpha=q$ form a system of $q$ equations with precisely $q$ undetermined functions $\xi, \lambda_{1}^{q}, \ldots, \lambda_{q-1}^{q}$. Therefore, for each first order generalized symmetry, we can uniquely determine these functions so as to satisfy the tangential contact conditions; the remaining normal contact conditions will then follow automatically from the symmetry conditions. Thus for codimension 1 systems satisfying the descent property, there is a one-to-one correspondence between first order generalized symmetries and internal symmetries. More generally, for a system of codimension $c>1$, the tangential contact conditions (33) form a system of $q c$ equations involving $c(q-c)+1$ undetermined functions, so there will be $c^{2}-1$ additional equations a first order generalized symmetry must satisfy in order that it correspond to an internal symmetry. For instance, any first order generalized symmetry of a codimension 2 system must satisfy 3 additional constraints for it to be an internal symmetry. For example, the codimension 2 equation $v_{x} u_{x x}=w$ has the first order generalized symmetry $\mathbf{v}=x^{2} \partial_{u}+2 v_{x} \partial_{w}$, but there is no internal counterpart, since it does not satisfy the tangential contact conditions (33).

The most important example of a codimension 1 system is the under-determined ordinary differential equation

$$
v_{x}=\left(u_{x x}\right)^{2} .
$$

Equation (34) was introduced by Hilbert, [6], as an example of an under-determined differential equation whose general solution could not be expressed in terms of an arbitrary function and a finite number of its derivatives. Subsequently, Cartan, $[\mathbf{2}],[\mathbf{3}]$, proved that this equation has the 14 dimensional exceptional Lie group $G_{2}$ as an internal symmetry group. Cartan's result can be verified directly by computing the first order generalized symmetries using the standard algorithm, and then invoking the preceding remarks to determine the corresponding internal symmetries. 
Theorem 11. Every first order generalized symmetry of the Hilbert-Cartan equation is a linear constant coefficient combination of the following fourteen generalized vector fields

$$
\begin{aligned}
\mathbf{v}_{1}= & \partial_{u} \\
\mathbf{v}_{2}= & \partial_{v}, \\
\mathbf{v}_{3}= & x \partial_{u}, \\
\mathbf{v}_{4}= & x^{2} \partial_{u}+4 u_{x} \partial_{v}, \\
\mathbf{v}_{5}= & x^{3} \partial_{u}+12\left(x u_{x}-u\right) \partial_{v}, \\
\mathbf{v}_{6}= & u \partial_{u}+2 v \partial_{v}, \\
\mathbf{v}_{7}= & 3 v \partial_{u}+4 v_{x}^{3 / 2} \partial_{v}, \\
\mathbf{v}_{8}= & u_{x} \partial_{u}+v_{x} \partial_{v}, \\
\mathbf{v}_{9}= & \left(2 x u_{x}-3 u\right) \partial_{u}+2 x v_{x} \partial_{v}, \\
\mathbf{v}_{10}= & \left(x^{2} u_{x}-3 x u\right) \partial_{u}+\left(x^{2} v_{x}-4 u_{x}^{2}\right) \partial_{v}, \\
\mathbf{v}_{11}= & \left(3 x v-4 u_{x}^{2}\right) \partial_{u}+\left(4 x v_{x}^{3 / 2}-8 u_{x} v_{x}\right) \partial_{v}, \\
\mathbf{v}_{12}= & \left(3 x^{2} v-8 x u_{x}^{2}+12 u u_{x}\right) \partial_{u}+\left(12 u_{x} v+12 u v_{x}+4 x^{2} v_{x}^{3 / 2}-16 x u_{x} v_{x}\right) \partial_{v}, \\
\mathbf{v}_{13}= & \left(3 x^{3} v-12 x^{2} u_{x}^{2}+36 x u u_{x}-36 u^{2}\right) \partial_{u}+ \\
& \quad+\left(36 x u_{x} v-36 u v+4 x^{3} v_{x}^{3 / 2}-24 x^{2} u_{x} v_{x}+36 x u v_{x}-16 u_{x}^{3}\right) \partial_{v}, \\
\mathbf{v}_{14}= & \left(9 u v-4 u_{x}^{3}\right) \partial_{u}+\left(9 v^{2}-12 u_{x}^{2} v_{x}+12 u v_{x}^{3 / 2}\right) \partial_{v} .
\end{aligned}
$$

According to (16), (31), any first order generalized symmetry

$$
\mathbf{v}_{Q}=Q\left(x, u, u_{x}, v, v_{x}\right) \partial_{u}+R\left(x, u, u_{x}, v, v_{x}\right) \partial_{v}
$$

of a codimension one system of the form

$$
u_{x x}=F\left(x, u, u_{x}, v, v_{x}, v_{x x}\right),
$$

is equivalent to the internal symmetry $\mathbf{X}=\operatorname{pr}^{(2)} \mathbf{v} \mid \mathcal{R}$, where $\mathbf{v}=\xi \partial_{x}+\varphi \partial_{u}+\psi \partial_{v}$ has

$$
\xi=-R_{u_{x}} F_{v_{x x}}-R_{v_{x}}, \quad \varphi=Q+\xi u_{x}, \quad \psi=R+\xi v_{x} .
$$

Thus, for example, the internal symmetry equivalent to $\mathbf{v}_{7}$ is given by

$$
\mathbf{X}_{7}=-6 v_{x}^{1 / 2} \partial_{x}+\left(3 v-6 u_{x} v_{x}^{1 / 2}\right) \partial_{u}-2 v_{x}^{3 / 2} \partial_{v}-3 v_{x} \partial_{u_{x}}+3 v_{x}^{-1 / 2} v_{x x}^{2} \partial_{v_{x x}}
$$

Note that, according to (38), the six symmetries $\mathbf{v}_{1}, \mathbf{v}_{2}, \mathbf{v}_{3}, \mathbf{v}_{6}, \mathbf{v}_{8}, \mathbf{v}_{9}$ are found to be equivalent to point symmetries, while the remaining eight are true internal symmetries. Since each of the vector fields in Theorem 11 corresponds to a unique internal symmetry, we deduce that these vector fields close to form a Lie algebra when restricted to the equation. Using standard Lie-algebraic techniques (Killing form, Cartan subalgebra, root diagrams, etc.), it can be proven that this Lie algebra is isomorphic to the non-compact real form of Lie algebra for the exceptional simple Lie group $G_{2}$. Therefore, we obtain Cartan's explicit realization of $G_{2}$ as the group of internal symmetry transformations of the six-dimensional manifold defined by the Hilbert-Cartan equation. 
Interestingly, there are additional higher order generalized symmetries of the HilbertCartan equation. An explicit example is the third order symmetry

$$
\mathbf{v}=u_{x x x} \partial_{u}+\left(2 u_{x x} u_{x x x x}-u_{x x x}^{2}\right) \partial_{v} .
$$

The full structure of the generalized symmetries of the Hilbert-Cartan equation and various generalizations has been determined by P. Kersten, [8], [9].

Acknowledgments. Besides my collaborators, Ian Anderson and Niky Kamran, without whom this work would not have come to fruition, I would like to thank Paul Kersten for checking our symmetry group computations, and George Bluman, who raised a question during my lecture in Catania that prompted us to properly formulate the descent property.

\section{References}

[1] Anderson, I.M., Kamran, N., and Olver, P.J., Internal, external and generalized symmetries, Adv. in Math., to appear.

[2] Cartan, É., Sur l'équivalence absolue de certains systèmes d'équations différentielles et sur certaines familles de courbes, in: Oeuvres Complétes, part. II, vol. 2, Gauthiers-Villars, Paris, 1953, pp. 1133-1168.

[3] Cartan, É., Sur l'intégration de certains systèmes indéterminés d'équations différentielles, in: Oeuvres Complétes, part. II, vol. 2, Gauthiers-Villars, Paris, 1953, pp. 1169-1174.

[4] Champagne, B., Hereman, W., and Winternitz, P., The computer calculation of Lie point symmetries of large systems of differential equations, Comp. Phys. Comm. 66 (1991), 319-340.

[5] Hereman, W., Review of symbolic software for the calculation of Lie symmetries of differential equations, Euromath Bull. 2 (1993), to appear.

[6] Hilbert, D., Über den Begriff der Klasse von Differentialgleichungen, in: Gesammelte Abhandlungen, vol. 3, Springer-Verlag, Berlin, 1935, pp. 81-93.

[7] Ibragimov, N.H., Transformation Groups Applied to Mathematical Physics, D. Reidel, Boston, 1985.

[8] Kersten, P.H.M., The general symmetry algebra structure of the underdetermined equation $u_{x}=\left(v_{x x}\right)^{2}$, J. Math. Phys. 32 (1991), 2043-2050.

[9] Kersten, P.H.M., The Lie-Bäcklund algebra structure for the general underdetermined equation $u_{r}=F\left(x, u, \ldots, u_{r-1}, v, \ldots, v_{k}\right)$, Nonlinearity 5 (1992), 763-770.

[10] Krasil'shchik, I.S., Lychagin, V.V., and Vinogradov, A.M., Geometry of Jet Spaces and Nonlinear Partial Differential Equations, Gordon and Breach, New York, 1986.

[11] Noether, E., Invariante Variationsprobleme, Nachr. Konig. Gesell. Wissen. Gottingen, Math.-Phys. Kl. (1918), 235-257.

[12] Olver, P.J., Applications of Lie Groups to Differential Equations, Graduate Texts in Mathematics, vol. 107, Springer-Verlag, New York, 1986. 